\title{
Vulnerabilidade socioambiental, redução de riscos de desastres e construção da resiliência - lições do terremoto no Haiti e das chuvas fortes na Região Serrana, Brasil
}

\author{
Socio-environmental vulnerability, disaster risk-reduction \\ and resilience-building - lessons from the earthquake in Haiti \\ and torrential rains in the mountain range close to Rio de Janeiro \\ in Brazil
}

Carlos Machado de Freitas ${ }^{1}$

Mauren Lopes de Carvalho ${ }^{2}$

Elisa Francioli Ximenes ${ }^{2}$

Eduardo Fonseca Arraes ${ }^{2}$

José Orlando Gomes ${ }^{3}$

${ }^{1}$ Centro de Estudos da Saúde do Trabalhador e

Ecologia Humana, Escola Nacional de Saúde Pública, Fundação Oswaldo Cruz. Rua Leopoldo Bulhões 1480/ 503, Manguinhos.

21.041-210 Rio de Janeiro

RJ.carlosmf@ensp.fiocruz.br

${ }^{2}$ Centro de Estudos e

Pesquisas de Emergências e

Desastres em Saúde,

Fundação Oswaldo Cruz

${ }^{3}$ Programa de Pós-

Graduação em Informática,

Departamento de

Engenharia Industrial,

Escola Politécnica,

Universidade Federal do

Rio de Janeiro.

\begin{abstract}
Data on disasters around the world reveal greater seriousness in countries with lower social and economic development levels. In this context, disaster risk-reduction and resiliencebuilding policies are priorities in the sustainable development agenda, featuring among the topics selected for the Rio+20 Summit. By means of a contribution of a conceptual nature and from examples of disasters in countries with different development levels, namely the Haiti earthquake and the torrential rains in the mountain range close to Rio de Janeiro in Brazil, the scope of this article is to demonstrate how socio-environmental vulnerability creates conditions for disasters, while at the same time limiting strategies for their prevention and mitigation. Lastly, some of the measures that disaster risk reduction and resilience-building demand in a socio-environmental vulnerability context are highlighted. These involve changes in the current patterns of social, economic and environmental development geared toward ecological sustainability and social justice as pillars of sustainable development.
\end{abstract}

Key words Natural disasters, Socio-environmental vulnerability, Risk reduction, Resiliencebuilding
Resumo Dados sobre desastres no mundo apontam para uma maior gravidade nos países com menores níveis de desenvolvimento econômico e social. Neste contexto, políticas de redução de riscos de desastres e construção da resiliência constituem prioridades na agenda do desenvolvimento sustentável, estando entre os temas eleitos para a Rio +20 . O objetivo deste artigo é, através de uma contribuição de natureza conceitual e dos exemplos de desastres em países com níveis de desenvolvimento diferentes, o terremoto do Haiti e as chuvas fortes na Região Serrana (Rio de Janeiro, Brasil), demonstrar como a vulnerabilidade socioambiental cria condições para os desastres, ao mesmo tempo em que limita as estratégias para prevenção e mitigação. Ao final são apontados alguns dos desafios que a redução de riscos de desastres e a construção da resiliência exigem em contextos de vulnerabilidade socioambiental, o que inclui mudanças nos padrões de desenvolvimento social, econômico e ambiental orientados para a sustentabilidade ecológica e a justiça social como pilares do desenvolvimento sustentável.

Palavras-chave Desastres naturais, Vulnerabilidade socioambiental, Redução de riscos, Resiliência 


\section{Introdução}

A redução de riscos de desastres e a construção da resiliência estão entre os temas eleitos pelo Secretariado da Conferência das Nações Unidas sobre Desenvolvimento Sustentável, conhecida como Rio +20 . Isto porque se nenhum país se encontra imune aos desastres, independente do seu nível de desenvolvimento econômico e social, os dados demonstram que a vulnerabilidade das sociedades ou comunidades é estreitamente relacionada ao nível de desenvolvimento.

Nos últimos 40 anos, mais de 3,3 milhões de óbitos por desastres estiveram concentrados nos países mais pobres ${ }^{1}$, e a cada ano, cerca de 226 milhões de pessoas são afetadas pelos mesmos. Entre 2000 e 2010, 680 mil pessoas foram vítimas fatais de terremotos e quase metade destes no ocorrido no Haiti. Em média 102 milhões de pessoas são afetados por enchentes a cada ano no mundo, 37 milhões por ciclones, furacões e tufões e 366 mil por deslizamentos de terra. Secas e estiagens, encontram-se associadas à perda de 558 mil vidas e afetam 1,6 bilhões de pessoas no mundo desde 1980. Se estes eventos impactam de modo mais grave a saúde das populações nos países mais pobres, é importante observar que alguns grupos populacionais encontram-se ainda mais vulneráveis, como mulheres e crianças, que possuem 14 vezes mais chances de óbito em um desastre ${ }^{2}$.

Só entre 2000 e 2010 as consequências econômicas dos desastres alcançaram um trilhão de dólares. Estes altos custos contribuem para que 1/5 de toda assistência humanitária seja dedicada aos desastres; porém menos de um por cento é destinado às ações de redução de risco de desastres ${ }^{1}$. E, mesmo que ocorra aumento do percentual para a redução de risco de desastres na assistência humanitária internacional ou nos orçamentos governamentais, estes não serão suficientes sem mudanças nos padrões de desenvolvimento.

Considerando a importância do tema dos desastres para o Brasil e outros países da América Latina e Caribe (ALC), o objetivo deste artigo é apresentar uma contribuição de natureza conceitual acerca dos aspectos relacionados à vulnerabilidade socioambiental, à redução de riscos de desastres e à construção da resiliência nas suas relações com o desenvolvimento sustentável. Para tanto, no primeiro item recorre-se a um conjunto de conceitos que nos permitem compreender os desastres como expressões dos padrões de desenvolvimento, que combinam processos sociais, entre estes as iniquidades, e a degradação ambiental.

Na segunda parte são utilizados como exemplos dois tipos de desastres (intensivo e extensi- vo) distintos, terremoto e chuvas fortes, que ocorreram no mesmo dia, em anos distintos e em países com condições de vulnerabilidade socioambiental bastante diferentes. Para cada uma das realidades destes países da ALC os desastres utilizados como exemplos estão entre os mais graves já ocorridos. Ao final é proposto que a redução de riscos de desastres e a construção da resiliência, um dos temas da Rio+20, deve envolver um conjunto de ações para a redução da vulnerabilidade socioambiental diretamente relacionadas à mudanças nos padrões de desenvolvimento social, econômico e ambiental vigentes.

\section{Desastres e padrões de desenvolvimento}

Como observado no título do relatório do Banco Mundial de 2011, Natural Hazards, UnNatural Disasters, se eventos geológicos como o terremoto do Haiti, e hidrometeorológicos, como chuvas fortes na Região Serrana podem ser considerados ameaças naturais, os desastres não são naturais ${ }^{1}$. São produzidos socialmente e a vulnerabilidade das sociedades ou comunidades encontra-se estreitamente e inversamente relacionada ao nível de desenvolvimento econômico e social ${ }^{3}$.

No âmbito da saúde pública, para que um evento se constitua em um desastre é necessário que combine alguns fatores que devem ser bem compreendidos. Primeiro, é necessário um evento detonador, conceituado como ameaça, que se relaciona a qualidade dos eventos físicos que podem ser gerados pela dinâmica da natureza (geológicas, hidrometeorológicas, biológicas) ou da sociedade (degradação ambiental ou ameaças tecnológicas como rompimentos de barragens, acidentes químicos e nucleares). Este evento deve resultar na exposição de populações humanas, gerando o potencial de danos e agravos à saúde. E estes serão mais ou menos graves a depender das condições de vulnerabilidade, que resultam tanto na propensão de uma comunidade ou sociedade de sofrer de modo mais intenso e grave os efeitos dos desastres, como também nas limitações das capacidades de redução de riscos e de resiliência frente a estes eventos ${ }^{4}$.

As condições de vulnerabilidade resultam de processos sociais e mudanças ambientais que é denominada de vulnerabilidade socioambiental, pois combinam: 1) os processos sociais relacionados à precariedade das condições de vida e proteção social (trabalho, renda, saúde e educação, assim como aspectos ligados à infraestrutura, como habitações saudáveis e seguras, estradas, saneamento, por exemplo) que tornam determinados grupos populacionais (por exemplo, mu- 
lheres e crianças), principalmente entre os mais pobres, vulneráveis aos desastres; 2) as mudanças ambientais resultantes da degradação ambiental (áreas de proteção ambiental ocupadas, desmatamento de encostas e leitos de rios, poluição de águas, solos e atmosfera, por exemplo) que tornam determinadas áreas mais vulneráveis quando da ocorrência de uma ameaça e seus eventos subsequentes. Em síntese, a vulnerabilidade socioambiental resulta de estruturas socioeconômicas que produzem simultaneamente condições de vida precárias e ambientes deteriorados, se expressando também como menor capacidade de redução de riscos e baixa resiliência ${ }^{4-7}$.

Se as perdas e os danos causados pelos desastres são crescentes, em condições de vulnerabilidade socioambiental não só afetam mais os países e populações mais pobres, como também ameaçam sua sobrevivência e os meios de vida, comprometendo os elementos básicos de sua dignidade e bem-estar (acesso aos alimentos, água de qualidade, habitação, bem como aos serviços, como educação e saúde) e trazendo riscos de inúmeros agravos e doenças. Integra um ciclo vicioso em que tanto se encontra na raiz dos desastres e agravamento de suas consequências, como na perda das capacidades de redução de riscos e construção da resiliência.

Dentre os desastres "naturais", os eventos de origem hidrometeorológica, constituem a grande maioria dos mesmos, principalmente tempestades e enchentes. Na ALC estes eventos correspondem a cerca de $70 \%$ do total e os de origem geológica, como os terremotos, a cerca de $20 \%{ }^{1}$.

Dados sobre enchentes e inundações no mundo revelam que há uma tendência de crescimento do número de pessoas expostas e afetadas por estes eventos, que se elevará para além das cerca de 102 milhões de pessoas por $a^{2}{ }^{2}$. Porém, embora enchentes e inundações constituam um problema global, dados de 2009 da Estratégia Internacional de Redução de Desastres ${ }^{8}$ revelam que $96 \%$ da população exposta a estes eventos no mundo e $95 \%$ dos óbitos encontram-se concentrados nos países com renda per capita menor do que 3.705 dólares por ano.

Situação similar também ocorre com outros tipos de desastres "naturais", como de origem geológica, caso dos terremotos. Resultaram em 680 mil vítimas fatais entre 2000 e 2010 , afetando principalmente populações que viviam em habitações precárias ${ }^{2}$. Dois exemplos se encontram na própria ALC, com os terremotos que atingiram o Haiti e o Chile. Embora o terremoto de fevereiro de 2010 no Chile (renda per capita de aproximadamente 9.800 dólares por ano) tenha sido mais forte do que o ocorrido em janeiro de 2011 no Haiti (renda per capita de 650 dólares por ano) ${ }^{9,10}$, o número de vítimas fatais foi de 385 à 519 vezes maior neste segundo, a depender dos dados oficiais que se considera. O terremoto do Chile atingiu uma magnitude de 8.8 na escala Richter, com duração de 3 minutos ${ }^{9}$ e uma quantidade de energia liberada 500 vezes maior que do Haiti, resultando em 577 vítimas fatais. O terremoto do Haiti teve magnitude de 7.0 à 7.3, com duração de 35 segundos e resultou em 222.570 vítimas fatais na estimativa oficial de janeiro de 2010 e chegou a 300 mil na estimativa de janeiro 2011.

O terremoto do Haiti é um exemplo de desastres intensivos, que possuem baixa frequência, mas são geograficamente concentrados e com grande potencial de impactos. Já os desastres extensivos correspondem a $97 \%$ dos eventos relacionados ao clima, possuindo alta frequência, não causando números significativos de óbitos, mas sendo responsáveis por grande proporção de danos à infraestrutura local e às habitações e condições de vida das comunidades e sociedades de baixa renda. Porém, importante observar que riscos de desastres extensivos que acabam por se tornar "normalidade" para muitas sociedades e comunidades, podem se tornar desastres intensivos amanhã ${ }^{11}$, sendo um típico exemplo as chuvas fortes e os deslizamentos que atingiram a Região Serrana do Rio de Janeiro (Brasil) em janeiro de 2011.

Os desastres (intensivos ou extensivos) não existem em um vácuo. São constituídos socialmente através de processos que se estruturam na dinâmica do desenvolvimento econômico e social, bem como da proteção social e ambiental ${ }^{4}$. Para melhor compreende-los como expressões dos padrões de desenvolvimento, a seguir estão os exemplos dos desastres do Haiti e da Região Serrana (RJ, Brasil).

\section{Vulnerabilidade socioambiental e desastres - os exemplos do Haiti e da Região Serrana}

No dia 12 de janeiro, em anos distintos, Haiti (2010) e Brasil (2011) sofreram seus maiores desastres em termos de óbitos, além dos impactos sociais e econômicos. O primeiro possui a população mais pobre da ALC em termos de PIB per capita. O segundo é o mais rico da ALC em termos de PIB, ainda que muito desigual em termos sociais.

\section{2 de janeiro de 2010}

- Porto Príncipe, Haiti

Em 12 de Janeiro de 2010, um terremoto de magnitude entre 7.0 e 7.3 na escala Richter e du- 
ração de 35 segundos com epicentro cerca de 25 $\mathrm{km}$ da capital do país, Porto Príncipe, atingindo cidades em um raio de $45 \mathrm{~km}$. O horário do terremoto foi às $16: 53$, momento em que grande parte da população estava nas ruas, o que provavelmente diminuiu o número de óbitos. O terremoto atingiu o centro econômico, administrativo, político e populacional do país, concentrando $66 \%$ do PIB e $39 \%$ da população localizada na cidade de Porto Príncipe e seus entornos ${ }^{10}$. As consequências humanas foram imensas, sendo destruída boa parte da economia e da capacidade de governo em um país historicamente vulnerável aos desastres (Tabela 1).

Mais de 2 milhões de pessoas foram diretamente afetadas pelo terremoto, representando $15 \%$ da população do país. Segundo informações oficiais em janeiro de 2011, o número de óbitos chegou a 300 mil e outras centenas de milhares lesionadas. Cerca de 1,3 milhões de pessoas passaram a viver em abrigos e outras $500 \mathrm{mil}$ se deslocaram para outras áreas do Haiti, exacerbando mais ainda problemas existentes de acesso a alimentos e serviços básicos. Por volta de 105 mil casas foram completamente destruídas e 208 mil danificadas. Em torno de 1.300 estabelecimentos educacionais e mais de 84 hospitais e centros de saúde foram severamente danificados ou destruídos. Boa parte do porto da capital ficou destruída, assim como prédios importantes do governo e da administração públi$\mathrm{ca}^{10}$. A precária situação econômica e social, combinada com a já imensa degradação ambiental no Haiti, resultou em uma condição de alta vulnerabilidade socioambiental e ausência de capacidade de redução do risco diretamente acoplado à precária capacidade de governo no país, agravando ainda mais os problemas sociais e econômicos, ambientais e sanitários.

A situação política, econômica, social e ambiental do Haiti precedente ao terremoto de 2010 já era bastante vulnerável, sendo o país mais pobre da ALC. As condições de vida eram bastante precárias para grande parte da população, com índi- ces de desnutrição próximos a uma emergência humanitária. Se aproximava de um desastre social crônico, resultando nos piores indicadores sociais (IDH 0.45 , considerado baixo $)^{9,10}$ e de saúde da ALC (mortalidade em menores de 5 anos de 76 por mil e esperança de vida ao nascer de 61,5 anos $)^{12}$.

No que se refere à situação ambiental, o Haiti possui imenso desmatamento das planícies, morros e encostas, processo iniciado com os espanhóis e continuado pelos franceses e, após a independência, pelos fazendeiros, que ocuparam os vales férteis e expulsaram os camponeses para as matas mais íngremes ${ }^{13}$. O longo processo de exploração da madeira e do solo no Haiti tornou o país muito mais vulnerável a tempestades do que a República Dominicana, embora compartilhem a mesma ilha. Enquanto na Republica Dominicana a proporção da superfície coberta por florestas era de 28,4\% em 2005, no Haiti era de $3,8 \%$, mais de 7 vezes menor ${ }^{14}$. O desmatamento, combinado com a vulnerabilidade às tempestades e ciclones, exacerbou a deterioração ambiental do país afetando a produtividade de alimentos, que caiu 30\% só entre 1991 e 2002, aumentando a pobreza rural e levando a uma maior migração do campo para Porto Príncipe (que já não possuía capacidade de absorver mais populações e de oferecer serviços, alimentos e saneamento ambiental adequado para todos) ${ }^{10,13}$.

Em relação ao acesso aos serviços básicos de saneamento e saúde, o Haiti já vivia uma situação em que menos da metade da população possuía acesso a serviços de saúde, água e saneamento, assim mesmo de baixa qualidade. Em relação aos serviços de saúde, $47 \%$ dos haitianos não possuíam acesso a atenção básica e $75 \%$ da atenção à saúde era provida por ONGs ou grupos religiosos, com a maioria sem nenhum controle do Ministério da Saúde. Em relação ao saneamento ambiental adequado, o Haiti apresenta os mais baixos percentuais de população com acesso a água potável (58\%) e esgotamento sanitário (19\%), com 8 milhões de habitantes, do total de 10 de milhões, sem acesso adequado à

Tabela 1. Desastres naturais ocorridos no século XXI no Haiti

\begin{tabular}{lcrr}
\hline \multicolumn{1}{c}{ Evento } & $\begin{array}{c}\text { Impacto } \\
\text { no PIB }\end{array}$ & $\begin{array}{c}\text { População } \\
\text { afetada }\end{array}$ & Óbitos \\
\hline 2004: Furacão Jeanne & $7 \%$ & 300.000 & 5.000 \\
2007: Furacões Dean e Noel & $2 \%$ & 194.000 & 330 \\
2008: Tempestades tropicais Fay e Gustav e Furacão Ike & $15 \%$ & 1.000 .000 & 800 \\
2010: Terremoto & $100 \%$ & 2.000 .000 & 222.500 \\
\hline
\end{tabular}

Fonte: $\mathrm{PAHO} / \mathrm{WHO}^{15}$ 
água e/ou saneamento. Esta situação resultava em um quadro precário de saúde, com altas taxas de doenças transmissíveis e parasitárias ${ }^{10,12}$.

O ciclo pobreza alimentava o da degradação ambiental e estes se retroalimentavam em um ciclo vicioso, tornando a situação da vulnerabilidade socioambiental do país mais pobre da ALC um terreno propício para o maior desastre já ocorrido nos últimos tempos. Nas condições existentes no Haiti, o terremoto não só resultou em alto grau de exposição da população, ao ser concentrado na área mais populosa do país, e efeitos imediatos, mas também em subsequentes. As consequências imediatas estiveram relacionadas aos desabamentos e soterramentos advindos da queda de construções (casas e edifícios), ocasionando muitas fraturas expostas, esmagamentos (ossos, vértebras e medula espinhal), cortes, perfurações e lacerações, além de queimaduras resultantes de incêndios subsequentes. Estes agravos e lesões, por falta de um sistema de saúde estruturado, resultaram em infecções e por vezes em amputações ${ }^{10}$.

As consequências pós-desastre estiveram relacionadas principalmente a destruição da pobre infraestrutura do país, como a de saneamento ambiental, gerando a exposição a doenças infecciosas, principalmente entre crianças, tanto na área diretamente afetada pelo terremoto, como nas menos afetadas em que houve um grande deslocamento de populações pressionando mais ainda os serviços e o acesso a alimentos e água potável. A população desabrigada passou a viver em abrigos temporários, sendo que um ano depois 550 mil se encontravam em 802 campos ao redor do país ${ }^{15}$, muitos com precário fornecimento de alimentos e condições insalubres, expondo principalmente crianças e mulheres lactantes aos riscos de doenças e desnutrição. Além disto, a perda de habitações, familiares, amigos e vizinhos, combinada com a destruição da infraestrutura, serviços e símbolos e lugares importantes do país, bem como a ruptura da ordem social, resultaram em um processo de exposição continuada aos problemas de saúde mental que emergem nestes eventos. Se no primeiro momento houve massiva migração para outros departamentos, por conta da destruição da infraestrutura em Porto Príncipe, houve, em um segundo momento, um fluxo inverso. A massiva distribuição de bens e serviços nos abrigos temporários na área metropolitana da capital não só contribuiu para o retorno dos que migraram, mas também para a migração das populações mais pobres dos departamentos não afetados em busca de melhores oportunidades ${ }^{10}$.
O terremoto deixou, além dos custos humanos, uma perda financeira estimada em 7,8 bilhões de dólares, retardando o crescimento econômico do país em 10 anos. A vulnerabilidade socioambiental não só agrava as consequências dos desastres, mas prolonga os mesmos em um ciclo vicioso, perdurando a crise humanitário que se encontra o país e aumentando os riscos de eventos como furacões, inundações e chuvas fortes que são comuns no país gerem novos desastres.

\section{2 de janeiro de 2011}

\section{- Região Serrana - Rio de Janeiro, Brasil}

Na madrugada do dia 12 de janeiro de 2011 ocorreram fortes chuvas na região Serrana do Rio de Janeiro que resultaram no que vem sendo considerado como o maior desastre climático ocorrido no Brasil. Na subestação de Ypu, em Nova Friburgo, por exemplo, choveu 220 milímetros em 24h, sendo acima de 80 milímetros considerado situação de alerta para a Região ${ }^{16}$. Estas chuvas resultaram em enchentes e deslizamentos que atingiram áreas rurais e urbanas, comunidades de baixo e de alto poder aquisitivo, algumas ficando totalmente isoladas, destruindo prédios e habitações, infraestrutura pública (73 pontes destruídas e as principais vias de acesso foram totalmente ou parcialmente afetadas), estabelecimentos de saúde e escolas, comprometendo principalmente os serviços de abastecimento de água, energia elétrica e telefonia fixa. As principais atividades econômicas da região foram prejudicadas, como indústrias, pecuária, agricultura e comércio, sendo que nestas duas últimas os prejuízos econômicos foram de 270 milhões $^{17}$ e 469 milhões ${ }^{18}$. Em termos de consequências humanas foram registrados 918 óbitos, 8.795 desabrigados e 22.604 desalojados ( Tabela 2), resultando no impacto direto sobre a vida de mais de 32 mil habitantes, principalmente nos municípios de Petrópolis, Teresópolis e Nova Friburgo, com o total de sete municípios decretando estado de calamidade pública.

As características ambientais desta região, que combina montanhas e muitos rios, subsolo composto de rocha e pequena camada de terra, associada ao fato de já ter ocorrido muita extração de madeira nativa, desmatamento e implantação de florestas com espécies exóticas para fins industriais foram, ao longo dos anos, tornando a mesma propicia a ocorrência de deslizamentos ${ }^{19}$. Os processos de degradação ambiental e ocupação combinados com as características geológicas e hidrometeorológicas (alta pluviosidade), tornam a região vulnerável à ameaças naturais como os deslizamentos de terra e enchentes. Por 
Tabela 2. Consequências humanas em termos de desalojados, desabrigados e óbitos no desastre de 12 de janeiro de 2012 na região serrana.

\begin{tabular}{lccrr}
\hline \multicolumn{1}{c}{ Município } & Desalojados & Desabrigados & Óbitos & Total \\
\hline Nova Friburgo & 4.528 & 789 & 429 & 5.476 \\
Cordeiro & 17 & 26 & - & 43 \\
Macuco & 28 & 24 & - & 52 \\
Bom Jardim & 1.186 & 632 & 2 & 1.820 \\
São Sebastião do Alto & 32 & 75 & - & 107 \\
Santa Maria Madalena & 284 & 44 & - & 328 \\
Petrópolis (Itaipava) & 6.956 & 187 & 71 & 7.214 \\
S José do Vale do Rio Preto & 300 & 174 & 2 & 474 \\
Areal & - & 8 & - & 8 \\
Teresópolis & 9.110 & 6.727 & 392 & 16.229 \\
Sumidouro & 163 & 109 & 22 & 294 \\
Total & $\mathbf{2 2 . 6 0 4}$ & $\mathbf{8 . 7 9 5}$ & $\mathbf{9 1 8}$ & $\mathbf{3 2 . 3 1 7}$ \\
\hline
\end{tabular}

Fonte: dados obtidos através da Defesa Civil do Estado do Rio de Janeiro.

esta razão que dos 11 municípios atingidos pelo desastre de 12 de janeiro, cinco (Petrópolis, Sumidouro, Nova Friburgo, Teresópolis e Bom Jardim) se encontram entre os 251 mais vulneráveis aos deslizamentos no país ${ }^{20}$.

O rápido crescimento da população (nos municípios mais atingidos, entre 1950 e 2010 a população triplicou em Petrópolis, quadruplicou em Nova Friburgo e quintuplicou em Teresópolis $)^{19}$ e a sua distribuição desordenada pelo território determinou a ocupação de áreas de proteção ambiental, como margens de rios e encostas, tanto oficialmente pelo poder público (como escolas e estabelecimentos de saúde, por exemplo), como ilegalmente, ocorrendo pouca ou nenhuma ação do estado para conter este processo. Segundo relatório produzido pelo Ministério do Meio Ambiente para a análise do desastre da Região Serrana, do total de 657 deslizamentos analisados em uma área na cidade de Nova Friburgo, 92\% estavam associados a algum tipo de alteração antrópica na vegetação em áreas de elevada inclinação (morros, montes, montanhas e serra), além da ocupação de faixas menores que 30 metros em cada margem dos rios aumentando a vulnerabilidade aos desastres ${ }^{21}$. O próprio setor saúde contribuiu para aumentar esta vulnerabilidade e também se tornou impactado pela mesma. Levantamento realizado pelo Ministério da Saúde nos municípios de Bom Jardim, Nova Friburgo, São José do Vale do Rio Preto e Sumidouro, constatou que de 43 estabelecimentos de saúde, 35 (81\%) estavam localizados em áreas de risco a ameaças naturais ${ }^{22}$.

Como uma fratura exposta da sociedade, o desastre revelou como a Região Serrana apre- sentava problemas crônicos de construções inadequadas em áreas de riscos (margens de rios e encostas), drenagem de águas, acúmulo de lixo nas encostas e desmatamentos, além da urbanização não planejada e a falta de terrenos próprios para moradias seguras ${ }^{21}$, contribuindo para aumentar a vulnerabilidade socioambiental e converte-las em desastres, expondo e afetando de modo mais intenso os mais pobres. Diferentemente do Haiti, os indicadores sociais dos três municípios com maior número de óbitos da Região Serrana eram melhores, com Nova Friburgo e Petrópolis possuindo em 2000 IDH elevados ( 0,8 ou mais), PIB per capita em 2010 acima de 8 mil dólares e incidência de pobreza abaixo de $16 \%$. Teresópolis possuía IDH médio $(0,7)$ e PIB per capita mais baixo, em torno de 7 mil dólares e maior incidência de pobreza em 2003, com 21,9\%. As taxas de mortalidade infantil em 2010 variavam de 12,2 por mil em Nova Friburgo à 19,6 por mil em Petrópolis, mas todos possuíam esperança de vida ao nascer igual ou superior a 70 $\operatorname{anos}^{23,24}$. Entretanto, apesar do desastre ter atingido populações de alta e baixa renda, em Teresópolis, das 3.400 habitações atingidas, $65 \%$ pertenciam à população de baixa renda ${ }^{17}$.

O desastre de 12 de janeiro, embora o mais grave já registrado no país, não ocorreu de uma hora para outra, sendo antecedido de uma série de outros em anos anteriores, como sintomas de um desastre anunciado, como se pode ver no Quadro 1. A análise deste quadro permite constatar que se passou de desastres com centenas de óbitos nos anos 1980, para desastres com dezenas de óbitos a partir do século XXI, ou de uma situação de desastres intensivos para extensivos, 
que transformados em "normalidade" contribuíram para que em janeiro de 2011 ocorresse o mais grave desastre já registrado no país em termos de óbitos.

O desastre expôs os limites da sociedade brasileira nas capacidades de redução de riscos de desastres, afinal um ano depois, o exemplo da Região Serrana revela limites estruturais do país, que podem ser resumidos em poucos gastos para a prevenção, e o que é gasto sendo realizado de modo fragmentado e parcial, não considerando novos cenários de riscos de desastres. Estudo realizado pela Confederação Nacional de Municípios $^{25}$ revela que entre 2004 e 2009, os gastos com respostas aos desastres foram da ordem de 1,9 bilhões de reais, enquanto os com prevenção e preparação foram correspondentes a 145 milhões de reais, ou pouco mais de $7 \%$ do montante gasto em respostas. No governo estadual, a situação é ainda pior, já que um ano após o desastre da Região Serrana, o orçamento da Secretaria Estadual de Defesa Civil para o Programa de Prevenção de Desastres previsto no início de 2012 era de apenas $1,7 \%$ do total do orçamento destinado à mesma ${ }^{17}$.

No nível municipal, pesquisa realizada pós-desastre apontou como limitações para as capacidades de redução de riscos a baixa participação comunitária, a falta de articulação entre instituições, a insuficiência de cadastros e mapeamentos de risco, a insuficiência dos Planos Municipais de Redução de Risco (obsoletos e restritos a algumas áreas dos municípios) como vulnerabilidades institucionais e organizacionais presentes na região ${ }^{19}$.

Estas vulnerabilidades institucionais e organizacionais, que integram a vulnerabilidade social, atingem todos os níveis de governo e se manifestam em obras parcialmente iniciadas (de 75 pontes prometidas para reconstrução, apenas uma foi entregue) ou contemplando apenas um pequeno percentual dos pontos de riscos (de 777 pontos identificados, obras foram iniciadas em apenas 30$)^{26}$, iniciativa parcial para a preparação e alerta de comunidades (capacitações de comunidades sem sirenes e pontos de apoio não claramente definidos em todas, bem como previsão de instalação de radares metereológicos e pluviômetros somente em 2013), apesar dos esforços para realização de exercícios simulados e capacitação de agentes comunitários para a prevenção de desastres realizados pelas Defesas Civis municipais, estadual e federal27.

Também foi detectado que recursos públicos liberados para reconstrução após desastres ocorridos no passado são muitas vezes desviados ou aplicados indevidamente com obras estruturais de custos altos que não levam em consideração a gestão e planejamento a partir da macro-bacia hidrográfica da região, "solucionando" o problema localmente, mas de forma ineficaz ${ }^{19}$. Mesmo soluções emergenciais, como a construção de habitações para solucionar o problema dos quase 9 mil desabrigados ainda não haviam iniciado um ano depois, contribuindo para que muitos retornassem às suas antigas habitações em áreas de riscos, como no caso de Teresópolis em que das 2.200 habitações afetadas pelo desastre, cerca de 2 mil tiveram o retorno de seus moradores, contribuindo também para isto limites no aluguel social ${ }^{17}$.

O desastre da Região Serrana acelerou a criação de estruturas como o Centro Nacional de Monitoramento e Alerta de Desastres Naturais (CEMADEN) no Ministério da Ciência e Tecnologia, forças nacionais como a Força Tarefa de Apoio Técnico e Emergência na Secretaria Nacional de Defesa Civil do Ministério da Integração Nacional e da Força Nacional do SUS no Ministério da Saúde, além da reformulação das políticas públicas relacionadas ao tema com o Projeto de Lei que institui o Estatuto da Proteção Civil no país. Apesar destes esforços, estima-se que apenas metade dos municípios brasileiros tenha alguma estrutura de Defesa Civil e até 2011 apenas 500 Coordenadorias Municipais de Defesa

Quadro 1. Histórico de enchentes e deslizamentos na Região Serrana do RJ

\begin{tabular}{|l|l|l|}
\hline Ano & \multicolumn{1}{|c|}{ Eventos e Consequências } & \multicolumn{1}{c|}{ Municípios afetados } \\
\hline 1987 & Deslizamento com 282 óbitos & Petrópolis e Teresópolis \\
\hline 1988 & Enchentes com 227 óbitos e 2mil desabrigados & Friburgo, Petrópolis e Teresópolis \\
\hline 2000 & Enchentes com 5 óbitos & Friburgo, Petrópolis e Teresópolis \\
\hline 2001 & Chuvas fortes com 48 óbitos e 793 desabrigados & Petrópolis \\
\hline 2003 & Chuvas fortes com 33 óbitos & Petrópolis \\
\hline 2007 & Chuvas fortes com 23 óbitos & Friburgo, Sumidouro, Petrópolis e Teresópolis \\
\hline 2008 & Chuvas fortes com 10 óbitos & Petrópolis \\
\hline
\end{tabular}


Civil integravam o Sistema Nacional de Defesa Civil, com outras 900 em fase de negociação ${ }^{17}$.

Embora a vulnerabilidade socioambiental na Região Serrana e no Brasil não possa ser tratada do mesmo modo que no caso do Haiti, há elementos estruturais de nossa realidade que não só contribuem para que os maiores impactos recaiam sobre os grupos sociais mais vulneráveis, principalmente os mais pobres, mas também prolonguem suas consequências através de um ciclo vicioso, com ainda baixa e fragmentada capacidades de redução de riscos e construção da resiliência.

\section{Algumas lições dos exemplos do Haiti e da Região Serrana}

Ambos os exemplos permitem vislumbrar que mantidas as condições do atual modelo de desenvolvimento econômico e social de ambos os países esboça-se um futuro de crescentes ameaças e vulnerabilidades socioambientais resultando em desastres com potencial de afetar grandes contingentes da população. Em escala global, não só as populações mais pobres nos países pobres são mais suscetíveis, como fica evidente no exemplo do Haiti, mas também as mais pobres mesmo nos países mais ricos, como ficou demonstrado no caso do Furacão Katrina que em 2005 afetou Nova Orleans nos EUA.

Desastres como os do Haiti e da Região Serrana no Brasil evidenciam que as condições latentes de vulnerabilidade socioambiental também estão na raiz da baixa capacidade de governança para estratégias de redução de riscos e construção da resiliência. Aproximadamente 90\% dos países com forte capacidade de governança para desastres são os de alta renda. Em contraposição, os países de baixa (como Haiti) e médiabaixa (como Brasil) renda respondem por $95 \%$ dos países com baixa capacidade de governança.

$\mathrm{O}$ rápido crescimento econômico de muitos países, como o Brasil, tem contribuído para reduzir a pobreza e melhorar as condições de vida de milhões de pessoas. Paradoxalmente crescem, principalmente neste grupo de países, os riscos de maiores impactos, incluindo os econômicos, pois as capacidades de governança para a redução de riscos e a resiliência não se desenvolvem com a mesma rapidez que o crescimento econômico. Esta realidade também vem ocorrendo nos países de alta renda, como os da Organização para Cooperação e Desenvolvimento Econômico (OCDE) ${ }^{11}$.

Diante deste quadro, o tema das políticas de redução de riscos de desastres vem recebendo grande atenção na agenda do desenvolvimento sustentável global, uma vez que se reconhece que podem contribuir para acelerar o desenvolvimento, proteger os investimentos e reduzir a pobre$\mathrm{za}^{1}$. Assim, estas políticas devem envolver desde a preparação para as respostas aos desastres até a construção de sociedades e comunidades resilientes através dos processos de recuperação e reconstrução. Os exemplos do Haiti e da Região Serrana não deixam dúvidas sobre a urgência desta agenda.

\section{Considerações finais}

Os riscos de desastres se constituem socialmente através de processos que se estruturam na dinâmica do desenvolvimento econômico e social, bem como da proteção social e ambiental ${ }^{4}$. É sobre estes processos que devem se fundamentar os conceitos e as práticas que constituem as bases para redução de riscos de desastres e da vulnerabilidade socioambiental, bem como para a construção da resiliência. A redução de riscos de desastres deve combinar um conjunto de políticas que previnam a ocorrência e limitem (mitigação e preparação) as consequências (perdas e danos) oriundas dos desastres. Isto envolve um conjunto de estratégias para a construção da resiliência que tornem as sociedades e as comunidades aptas a desenvolverem as habilidades de responder adequadamente aos eventos, monitora-los, antecipa-los e aprender com os mesmos, fortalecendo sua capacidade de adaptação após os desastres, mantendo um nível aceitável de funcionamento e estrutura para restabelecer-se, recuperar-se e reconstituir-se, não só retornando a normalidade de sua vida "cotidiana", como também em condições ainda mais sustentáveis e seguras do que as anteriormente existentes ${ }^{2-4,28}$.

Considerando a vulnerabilidade socioambiental como aspecto central para compreensão dos desastres, não se pode considerar que as capacidades de redução de riscos e de resiliência podem ser descontextualizadas dos processos que as geram. A questão de desenvolver habilidades e fortalecer as capacidades para tornar-se resiliente envolve mudanças de padrões, desde os cognitivos (o modo como se interpreta o mundo ao redor e seus eventos) até as políticas e ações que resultam nos macro-determinantes sociais, econômicos e ambientais que resultam não só nos aspectos básicos do viver (acesso ao trabalho, renda, alimentação, educação, saúde, habitação, saneamento ambiental, entre outros), como também de onde se vive e trabalha (uso e ocupação do solo, de gestão ambiental e apropriação dos recursos naturais, entre outros) articulados e in- 
tegrados com políticas sistêmicas orientadas para a sustentabilidade ecológica e a justiça social como pilares do desenvolvimento sustentável.

No caso do Haiti, deve-se reduzir a vulnerabilidade da população às ameaças fortalecendo a resiliência do país. Isso significa um processo de longuíssimo prazo de desenvolvimento econômico e social de forma sustentável. O Haiti deve pautar seu desenvolvimento na ideia de sustentabilidade, beneficiando os pobres ao mesmo tempo em que protege seus recursos naturais. $\mathrm{O}$ desastre de 2011 oferece uma oportunidade para que o Haiti não se reconstrua para a situação anterior ao desastre, mas orientado para um novo modelo que combine a preservação dos recursos naturais, o fortalecimento das instituições públicas de saúde e segurança, investimentos em infraestrutura e melhoria das habitações e condições de vida da população.

Para a região Serrana no Rio de Janeiro, em primeiro lugar é necessária a compreensão, por parte dos gestores (do nível federal ao local) e da população, de que existe uma vulnerabilidade climática e geofísica aos deslizamentos de terra e formação de enchentes. Em outras palavras, é necessário o reconhecimento que ameaças naturais e potenciais de desastres na região não são eventos inesperados. Esta compreensão deve nortear de forma urgente e necessária um redesenho organizacional do modo como o estado brasileiro enfrenta o tema dos desastres, de forma a buscar uma interoperalibilidade de sua estrutura visando um incremento da resiliência e gerando políticas integradas ${ }^{4,29}$. Desde políticas de prevenção da situação existente envolvendo políticas e planejamento da ocupação e uso do solo, incluindo as habitações e a exploração agrícola, até as de reconstrução de estradas, pontes, escolas e estabelecimentos de saúde, assim como as ações de preparação e respostas para desastres, devem ser integradas às políticas de desenvolvimento locais e regionais, atuando com transparência e participação da sociedade nos determinantes sociais e ambientais da saúde e na redução da vulnerabilidade socioambiental.

Resguardadas as diferenças nos níveis de vulnerabilidade socioambiental, no Haiti e no Brasil constata-se que ainda há um longo percurso para que o setor saúde atue mais diretamente na redução de riscos de desastres e construção da resiliência, inclusive reduzindo sua própria vulnerabilidade ao não estar preparado e ao mesmo tempo situado em áreas de riscos. Porém, com certeza este longo caminho só poderá ser integralmente percorrido com mudanças profundas no modelo de desenvolvimento, combinando políticas e ações que atuem sobre seus elementos estruturais, com mudanças sociais e culturais, do nível macro ao micro, atuando permanentemente sobre os determinantes da vulnerabilidade socioambiental para promover a saúde, a sustentabilidade ecológica e a justiça social.

\section{Colaboradores}

CM Freitas, ML Carvalho, EF Ximenes, EF Arraes e JO Gomes participaram igualmente de todas as etapas de elaboração do artigo.

\section{Referências}

1. World Bank (WB), United Nations (UN). Natural hazards, unnatural disasters: the economics of effective prevention. Washington DC: WB/UN; 2010.

2. United Nations Conference on Sustainable Development (UNCSD). Disaster Risk Reduction and Resilience Building. [documento da internet]. 2012 [acessado 2012 jan 12], [4 p.]. Disponível em: http:// www.uncsd2012.org/rio20/index.php?page $=$ view $\& n r=225 \&$ type $=400 \&$ menu $=45$

3. United Nations Conference on Sustainable Development (UNCSD). Disaster-resilient Societies - Facts and figures. [documento da internet]. 2012 [acessado 2012 jan 12]. [cerca de 2 p.]. Disponível em: http:// www.un.org/en/sustainablefuture/disasters.shtml

4. Narváez L, Lavell A, Ortega GP. La gestión del riesgo de desastres: un enfoque basado en procesos. San Isidro: Secretaría General de la Comunidad Andina; 2009. 
5. Alves HPF, Alves CD, Pereira MN, Monteiro AMV. Dinâmicas de urbanização na hiperperiferia da metrópole de São Paulo: análise dos processos de expansão urbana e das situações de vulnerabilidade socioambiental em escala intraurbana. Rev. bras. estud. Popul. 2010; 27(1):141-159.

6. Marandola Jr E, Hogan DJ. Vulnerabilidade do lugar vs. vulnerabilidade sociodemográfica: implicações metodológicas de uma velha questão. Rev. bras. estud. Popul. 2009; 26(2):161-181.

7. Natenzon C. Vulnerabilidad, incertitumbre y planificación participativa de desastres: el caso de las inundaciones catastróficas en Argentina. In: Porto MFS, Freitas CM, organizadores. Problemas ambientais e vulnerabilidade: abordagens integradoras para $o$ campo da saúde pública. Rio de Janeiro: Cesteh, Ensp, Fiocruz; 2002. p. 57-78.

8. International Strategy for Disaster Reduction (ISDR). Global Assessment Report on Disaster Risk Reduction - Risk and poverty in a changing climate Invest today for a safer tomorrow. Geneva: United Nations; 2009.

9. Organización Panamericana de la Salud (OPAS/ OMS). El terremoto y tsunami del 27 de febrero en Chile. Crónica y lecciones aprendidas en el sector salud. Santiago de Chile: OPS; 2010

10. Pan American Health Organization (PAHO). Health response to the earthquake in Haiti January 2010: Lessons to be learned for the next massive suddenonset disaster. Washington DC: PAHO; 2011.

11. International Strategy for Disaster Reduction (ISDR). Global Assessment Report on Disaster Risk Reduction - Revealing risk, redefining development. Geneva: United Nations; 2011.

12. Organización Panamericana de la Salud (OPAS/ OMS). Situación de salud en las américas - Indicadores Básicos. Washington DC: OPAS; 2009.

13. Programa de las Naciones Unidas para el Medio Ambiente (PNUMA). Informe sobre la Iniciativa Latinoamericana y Caribeña para el Desarrollo Sostenible (ILAC) a cinco años de su adopción - XVI Reunión del Foro de Ministros de Medio Ambiente de América Latina y el Caribe Santo Domingo, República Dominicana 27 de enero al 1 de febrero de 2008. Santo Domingo: PNUMA, Oficina Regional para América Latina y el Caribe; 2008.

14. Bourne Junior J. Pobre chão - Haiti perdeu o seu solo e os meios para recupera-lo. National Geographic Brasil 2008; setembro:78-81.

15. Pan American Health Organization (PAHO). Earthquake in Haiti - One Year Later. Washington: PAHO/WHO Report on the Health Situation; 2011

16. Instituto Estadual do Ambiente (INEA). Sistema de alerta de cheias. [documento da internet]. 2011, 12 jan. [acessado 2012 mar 20]; [cerca de 1p.]. Disponível em: http://inea.infoper.net/inea/?p=historico \&sen $=$ CHUVAINTERVALO\&leg $=$ COTA\&es $=2217$ 42310\& $\mathrm{dh}=1294797600000 \& \mathrm{df}=1294884000000$

17. A vida de Brayan. Jornal O Globo (Caderno Especial) $2012 ; 8$ jan. p.7.
18. Prejuízo de empresários da Região Serrana é de meio bilhão de reais. Jornal do Brasil digital 2011; 8 fev. [acessado 2012 jan. 25]. Disponível em: http:// www.jb.com.br/tragedia-na-serra/noticias/2011/02/ 08/prejuizo-de-empresarios-da-regiao-serrana-ede-meio-bilhao-de-reais/

19. Centro Universitário de Estudos e Pesquisas sobre Desastres da Universidade Federal de Santa Catarina. Diretrizes em Redução de Riscos de Desastres Região Serrana do Rio de Janeiro. Florianópolis: CEPED-UFSC; 2011.

20. Brasil. Ministério das Minas e Energia (MME). Seleção dos municípios críticos a deslizamentos - nota explicativa. Brasília: Serviço Geológico do Brasil / Secretaria de Geologia, Mineração e Transformação Mineral; 2011.

21. Brasil. Ministério do Meio Ambiente. Relatório de Inspeção Área atingida pela tragédia das chuvas Região Serrana do Rio de Janeiro. Brasília: Secretaria de Biodiversidade e Florestas; 2011.

22. Brasil. Ministério da Saúde (MS). Relatório Final Sala de Situação para atendimento ao Rio de Janeiro. Brasília: Secretaria de Vigilância em Saúde; 2011.

23. Fundação Centro Estadual de Estatísticas, Pesquisas e Formação de Servidores Públicos do Rio de Janeiro (CEPERJ). Anuário online [banco de dados da internet]. 2012. [acessado $2012 \mathrm{fev} 2$ ]. Disponível em: http://www.fesp.rj.gov.br/ceep/ent/anu online.html

24. Instituto Brasileiro de Geografia e Estatísticas (IBGE). IBGE Cidades. [banco de dados da internet]. 2012. [acessado 2012 fev 4]. Disponível em: http://www.ibge.gov.br/cidadesat/topwindow.htm?1

25. Confederação Nacional dos Municípios (CNM). Desastres naturais no Brasil - análise da portarias de situação de emergência e estado de calamidade pública de 2003 a 2010. (Estudo técnico CNM). Brasília: CNM; 2010.

26. Estado diz que precisa de mais $\mathrm{R} \$ 1$ bi para encostas. Jornal O Globo 2012; 12 jan. p. 14.

27. Região Serrana participa de simulado de preparação para desastres. Jornal do Brasil digital 2011; 15 out. [acessado 2012 jan 29]. Disponível em: http://www. jb.com.br/rio/noticias/2011/10/15/regiao-serranaparticipa-de-simulado-de-preparacao-para-desastres/

28. Hollnagel E, Pariès J, Woods D, Wreathal J. Resilience Engineering in Practice. Burlington: Ashgate; 2010.

29. Catalan C, Benoît R. Evaluation of organizacional resilience: application in Quebec. Proceedings of Fourth Resilience Engineering Symposium: Sophie Antipolis; Transvalor-Presses des Mines; 2011.

Artigo apresentado em 04/04/2012

Aprovado em 10/04/2012

Versão final apresentada em 15/04/2012 